\title{
Combating Coevolutionary Disengagement by Reducing Parasite Virulence
}

\author{
John Cartlidge \\ johnc@comp.leeds.ac.uk \\ Informatics Network, School of Computing, University of Leeds, LS2 9JT, UK \\ Seth Bullock \\ seth@comp.leeds.ac.uk \\ Informatics Network, School of Computing, University of Leeds, LS2 9JT, UK
}

\begin{abstract}
While standard evolutionary algorithms employ a static, absolute fitness metric, coevolutionary algorithms assess individuals by their performance relative to populations of opponents that are themselves evolving. Although this arrangement offers the possibility of avoiding long-standing difficulties such as premature convergence, it suffers from its own unique problems, cycling, over-focusing and disengagement.

Here, we introduce a novel technique for dealing with the third and least explored of these problems. Inspired by studies of natural host-parasite systems, we show that disengagement can be avoided by selecting for individuals that exhibit reduced levels of "virulence", rather than maximum ability to defeat coevolutionary adversaries. Experiments in both simple and complex domains are used to explain how this counterintuitive approach may be used to improve the success of coevolutionary algorithms.
\end{abstract}

Keywords

Coevolution, disengagement, host, parasite, virulence. 


\section{Introduction}

Coevolutionary disengagement occurs when one advantaged population outperforms another to the extent that conspecifics become indistinguishable from one another in terms of fitness. At such times, coevolving populations become decoupled, and selection acts indiscriminately causing the system to drift, often with deleterious results. This paper will explore the hypotheses that:

1. in order to successfully apply competitive coevolutionary algorithms to complex optimisation problems, it is necessary to ensure coevolutionary engagement, and that

2. a simple, general-purpose means of maintaining engagement is to manipulate fitness functions such that individuals in an advantaged population are selected to achieve moderate, rather than maximum, success.

We demonstrate that this "reduced virulence" technique tends to minimise the chance of coevolutionary disengagement by encouraging fitness diversity, and that as a result it improves coevolutionary optimisation in both simple and complex domains. Moreover, reducing virulence in this way is shown to influence the course of coevolutionary optimisation in a manner that differs from that achieved through standard diversity maintenance techniques such as competitive fitness sharing, or resource sharing.

This paper is organised as follows. Section 2 introduces coevolutionary algorithms and details the problem of disengagement. Section 3 introduces the reduced virulence technique and demonstrates its application in the context of a simple counting ones domain. Section 4 presents further results and analysis from the counting ones domain, including a comparison with an alternative method of maintaining engagement. Section 5 compares the reduced virulence technique to resource sharing, a standard method for maintaining population diversity, in the context of a simple matching game. In section 6, the performance of the reduced virulence technique is assessed against a more complex problem, coevolving minimum-length sorting networks. Finally, section 7 presents a brief discussion of the paper's results and future work.

\section{Coevolution}

\subsection{Background}

Standard evolutionary computation, often in the form of a genetic algorithm (Holland, 1975), has been used in a number of practical applications (see, e.g., Goldberg, 1989; Davis, 1991). Within a standard evolutionary framework, individuals receive a fitness score determined by a static function. This absolute fitness is objective and does not vary over time. However, effective absolute fitness measures are often difficult to define. To achieve success, an absolute fitness function must differentiate individuals throughout the evolutionary process and direct a population towards optimality. Choosing an adequate fitness function for a particular optimisation problem can prove to be as difficult as solving the optimisation problem itself.

Coevolutionary algorithms offer an alternative. Such algorithms determine the fitness of an individual in relation to others. As such, fitness is not static. There is no mapping between genotype and fitness that remains constant over time. Rather, the fitness of a genotype varies depending upon the make-up of other individualsrelative fitness. Measuring fitness in such a manner removes the necessity for defining 
an absolute function. As such, coevolutionary algorithms can be beneficial in domains when a suitable (absolute) fitness measure is difficult to determine.

Cooperative coevolutionary algorithms are often utilised in situations where a problem can be naturally decomposed into sub-components. Individuals represent such sub-components and are assessed in a series of collaborations with other individuals in order to form complete solutions (for example, Potter \& De Jong, 2000; Watson \& Pollack, 2000; Wiegand, Liles, \& De Jong, 2001). Cooperative coevolutionary algorithms have had success in a variety of domains, for example, manufacturing scheduling (Husbands \& Mill, 1991), function optimisation (Potter \& De Jong, 1994), designing artificial neural networks (Potter \& De Jong, 1995) and room painting (Puppala, Sen, \& Gordin, 1998).

Competitive coevolution either occurs within one population engaged in self-play, or between multiple populations. Individuals represent complete solutions that are gradually refined throughout an evolutionary run. Single population competitive coevolution has been successfully applied to the Iterated Prisoner's Dilemma (Axelrod, 1984; Lindgren \& Nordahl, 1994), pursuit and evasion (Reynolds, 1994), and to finding robust game strategies in, for example, Tic-Tac-Toe (Angeline \& Pollack, 1993), backgammon (Pollack, Blair, \& Land, 1996) and Texas Hold'em Poker (Noble \& Watson, 2001).

In this paper, we are interested in competitive coevolution between two populations (the simplest and most commonly used $\mathrm{N}$-population competitive algorithm) with inter-population assessment, often described as predator-prey, or host-parasite coevolution ${ }^{1}$. Such competitive coevolutionary algorithms have been successfully applied to discovering minimal-length sorting networks (Hillis, 1990; Juillé, 1995), finding CA rules to solve the density classification task (Juillé \& Pollack, 1998b, 1998a), designing artificial neural networks for robot control (Floreano \& Nolfi, 1997), pursuit and evasion (Cliff \& Miller, 1995), and the domains of 3-D Tic-Tac-Toe and Nim (Rosin \& Belew, 1997).

\subsection{Problems with Competitive Coevolution}

Competitive coevolutionary algorithms circumvent the problem of defining an absolute fitness function by utilising relative fitness assessment-individuals receive a score based upon their success against contemporary opponents. As relative fitness functions vary through time it is possible for a coevolutionary system to evolve in an unanticipated manner. Although we have had names for the problems associated with computational coevolution for some time, it is only recently that there has been a concerted effort to define them. In recent years there has been a drive to analyse the coevolutionary problems arising from relative fitness assessment (e.g., Watson \& Pollack, 2001; Bucci \& Pollack, 2002; Luke \& Wiegand, 2002), however, this work is still in its infancy.

In general, coevolutionary systems are difficult to direct. Individuals may over-fit their contemporary competitors, resulting in potentially brittle solutions that are unable to generalise (Watson \& Pollack, 2001; Bucci \& Pollack, 2002). Rather than enter a progressive arms-race, competing populations may stabilise at sub-optimal equilibria, or mediocre stable states (Angeline, 1994; Pollack et al., 1996; Juillé \& Pollack, 1998a; Ficici \& Pollack, 1998a; Pollack \& Blair, 1998; Bucci \& Pollack, 2002) whereby populations begin to collude-an example of this occurred in the trenches during World War I as soldiers on both sides refrained from attempting to kill the enemy (Axelrod, 1984). As individuals are only rewarded for out-performing their contemporary opponents, it is

\footnotetext{
${ }^{1}$ However, the approach explored here could be generalised to other coevolutionary frameworks.
} 
possible for earlier adaptations to be lost, potentially leading to cycling (Cliff \& Miller, 1995; Rosin, 1997; Shapiro, 1998; Juillé \& Pollack, 1998a; Ficici \& Pollack, 1998a; Bucci \& Pollack, 2002). Finally, if one population outperforms the other to the extent that every opponent is beaten, the gradient for selection disappears and the populations disengage and drift (Watson \& Pollack, 2001; Bucci \& Pollack, 2002; Cartlidge \& Bullock, 2002). As drift during periods of disengagement is random rather than neutral, above average populations are likely to degenerate (see section 6.4).

Although there are methods for counter-acting particular coevolutionary problems (e.g., fitness sharing and the "hall of fame", Rosin \& Belew, 1997), few of these address the problem of disengagement, the phenomenon that we are concerned with in this paper. In the following section disengagement is introduced in more detail.

\subsection{Disengagement}

Through the continuous feedback provided by relative fitness assessment, coevolving populations can be considered as a coupled dynamical system with each population evolving over a dynamic fitness landscape that continually fluctuates in response to perturbations from the other (see discussion of NKC landscapes in Kauffman \& Johnsen, 1991). It is this interactive dynamic that drives the selection pressure between populations, continually eroding the adaptive advantage of each population (the Red Queen Effect, van Valen, 1973) and potentially resulting in an evolutionary arms race (Dawkins \& Krebs, 1979). Disengagement occurs when a coevolutionary system decouples - each population no longer perturbs the other, thus not only eliminating feedback between the coevolving populations, but eradicating any means of relative fitness assessment-resulting in evolutionary drift.

Often, coevolutionary systems are asymmetric - hosts and parasites may differ genetically (in terms of encoding) or behaviourally (in terms of goal strategy). Such asymmetry may result in an inherent advantage for one population. When coevolving pursuers and evaders, for example, it is often much easier, at least initially, to be a successful evader (Cliff \& Miller, 1995). Given that disengagement results from one population out-performing the other, it is intuitive that an inherent asymmetrical advantage favouring a particular population will encourage coevolutionary disengagement.

Consider an asymmetrical host-parasite system in which parasites enjoy an inherent advantage. Let us assume that the system is nearing disengagement, with the majority of parasites scoring maximally in the majority of competitions against hosts. The few parasites that some hosts are able to beat-those that discriminate hostswill receive relatively low fitness and as such will have few progeny. In contrast, the parasites able to beat all current opponents-and thus unable to discriminate among hosts-receive high fitness, thus leaving many offspring. In such a situation, it is likely that subsequent parasite generations will tend to comprise increasing numbers of individuals capable of beating all current opponents-i.e., there will be less discrimination among hosts despite any genetic and phenotypic diversity. Eventually the populations will disengage, with every host achieving the same poor score, and every parasite achieving the same high score. At this point, both populations will drift.

Although disengagement has entered the terminology of coevolutionary computing relatively recently (Bucci \& Pollack, 2002; Cartlidge \& Bullock, 2002), the phenomenon has previously been recognised. Coevolutionary coupling, or engagement has been described as maintaining a gradient for selection with which to discriminate individuals (Watson \& Pollack, 2001), coevolving an ideal training set with which to supply feedback (Juillé \& Pollack, 1998a, 1998b), maintaining learnability (Ficici \& Pol- 
The Phantom Parasite

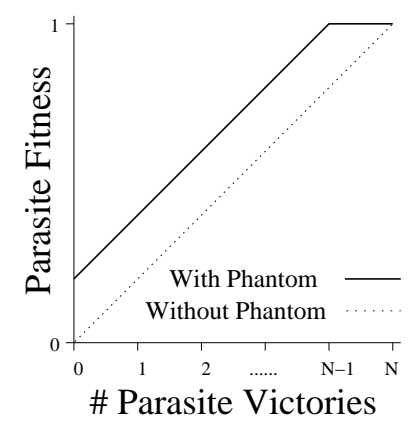

The $\Phi$ Function

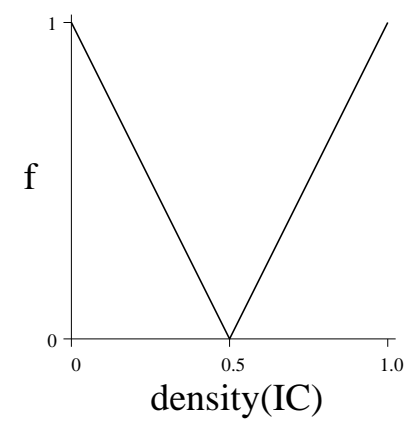

Figure 1: The phantom parasite function (left). Individuals that beat all real opponents lose to the phantom parasite. Individuals that lose to at least one real opponent beat the phantom parasite. The $\Phi$ function (right) - fitness of an IC is 0 if classified correctly, $f$ otherwise-produces a stabilising selection pressure favouring ICs that are neither too easy, nor too hard, to classify (see equation 1).

lack, 1998b, 1998a) or providing pedagogical stepping stones (Rosin, 1997). The decoupling of coevolutionary populations is problematic and as such there have been several attempts to produce a technique to circumvent it. Methods proposed to counter-act disengagement are discussed below.

The phantom parasite (Rosin, 1997) inhibits the reproductive influence of unbeatable individuals (figure 1, left). Used in conjunction with competitive fitness sharing (Rosin \& Belew, 1995), this anti-elitist innovation has no effect on individuals who achieve a less than perfect score. However, any individual that scores perfectly automatically has its score reduced slightly. This punishment is glossed in terms of interactions with an "ideal" phantom parasite: 'Hosts that lose to some current parasite defeat the phantom parasite. Hosts that defeat all current parasites lose to the phantom parasite' (Rosin, 1997). Effectively, the phantom parasite transforms the fitness function of parasites as shown in figure 1 , left. Individuals winning $N-1$ contests receive an equivalent score to those winning $N$ contests. The aim is to discourage parasites from performing too well, however, it remains in the best interests of an individual parasite to win as many competitions as possible.

The friendly competitor (Ficici \& Pollack, 1998a, 1998b) attempts to coerce a coevolutionary arms-race through utilising a three population system. A parasite population coevolves with two host populations, one friendly and one hostile. Parasites are rewarded for being an easy challenge for friendly hosts whilst simultaneously being a difficult challenge for hostile hosts. When coevolving sorting networks, for example, a parasite list would be rewarded for simultaneously being unsorted by hostile host networks and sorted by friendly host networks. In this way, parasites are punished for becoming too difficult for all hosts. The friendly competitor is designed to maintain a selection gradient for hosts, however, it is a complicated technique that requires the introduction of a third population and assumes that it is possible for parasites to simultaneously be both competitive and cooperative. To enable versatility, a simpler approach is required, such as that of the entropy measure, discussed below.

The entropy measure (Juillé \& Pollack, 1998a, 1998b), later adapted as Pagie's $\Phi$ function (Pagie \& Hogeweg, 2000; Pagie \& Mitchell, 2002), utilises domain-specific knowl- 
edge for the density classification task for cellular automata (CAs) to reduce disengagement in that specific domain (figure 1, right). The aim of the density classification task is to coevolve CA rules for classifying the density of an initial condition (IC) - a binary string-as greater (or less) than 0.5 depending upon whether the IC contains more (or less) than $50 \%$ 1s (see Mitchell, Crutchfield, \& Hraber, 1994). Having an inherent advantage, parasite initial conditions are known to become increasingly difficult to classify as their density approaches 0.5 . Thus, in order to encourage ICs to be challenging, without becoming too difficult, engagement is maintained by utilising the $\Phi$ function, where $\Phi(I C)$ gives the fitness of an initial condition (Pagie \& Mitchell, 2002).

$$
\Phi(I C)= \begin{cases}0 & \text { if classified correctly } \\ \mid \text { density }(I C)-\frac{1}{2} \mid & \text { otherwise }\end{cases}
$$

As parasite ICs are encouraged to be unclassifiable, it is likely that IC density will approach $50 \%$ - the most difficult to classify. However, the $\Phi$ function counteracts this by simultaneously rewarding ICs for deviating from $50 \%$ density. Thus, optimal ICs are as easy as possible to classify whilst still being unclassifiable. The $\Phi$ function performs well, but is domain-specific. Although Juillé and Pollack (1998a) state that they would like to produce heuristics to make this technique domain-general, both the entropy measure and the $\Phi$ function rely heavily upon domain-specific knowledge.

In summary, although disengagement is a recognised hindrance to coevolution, few techniques have been proposed to counteract disengagement, and those that have suffer from problems such as domain-specificity or lack of versatility. The only real domain-general solution that has been proposed is the Phantom Parasite, however, this has rarely been used. In the following section we introduce a novel technique for combating coevolutionary disengagement that is domain general, simple to implement and versatile.

\section{Reduced Parasite Virulence}

In this section we introduce a novel technique for combating disengagement, inspired by the effects of parasite virulence upon the dynamics of naturally occurring hostparasite systems.

\subsection{Natural Virulence}

Artificial coevolutionary systems are often described as analogous to natural predatorprey or host-parasite systems. Adopting Janzen's (1980) definitions, we can distinguish between true coevolutionary systems, which consist of two populations reciprocally adapting and counter-adapting to each other in a one-to-one relationship, and diffuse coevolutionary systems, which consist of multiple populations, each involved in a web of adaptations and counter-adaptations-a one-to-many relationship. In nature, true coevolution is only rarely observed, typically in host-parasite systems, whilst diffuse coevolution is frequently encountered in, for example, many predator-prey situations. Given that most coevolutionary algorithms employ only two populations, the host-parasite analogy is probably closer - one population (the parasite) is typically considered to pose problems for the other (the host) resulting in a series of adaptations and counter-adaptations that may result in an escalating coevolutionary armsrace (Dawkins \& Krebs, 1979).

Coevolutionary algorithms typically differ from natural systems in the way that they deal with parasite virulence (here defined as 'parasite-mediated morbidity and mortality in infected hosts' Levin, 1996). In order to ensure survival long enough to 

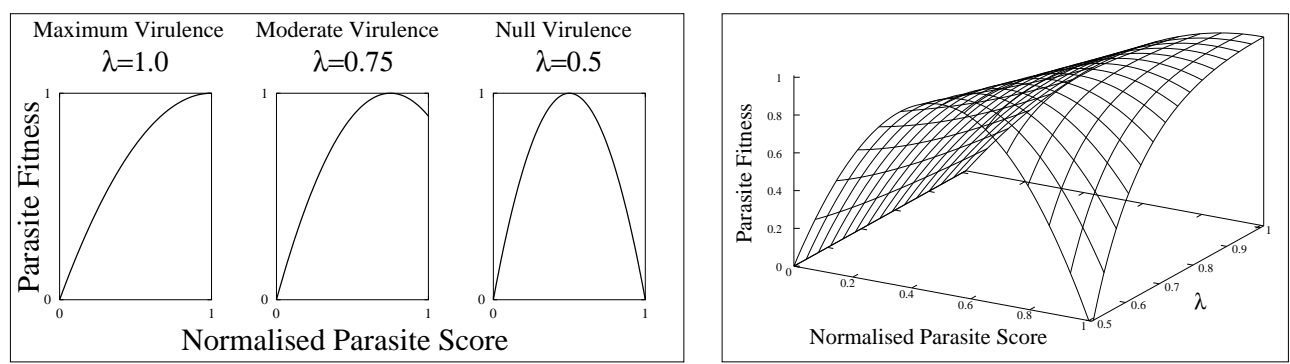

Figure 2: Parasite fitness is a function of both parasite score, $x$, and virulence parameter, $\lambda$, (see equation 2). For convenience, the labels Maximum, Moderate and Null virulence have been used for $\lambda$ values $1.0,0.75$ and 0.5 respectively (left), however, there exists a continuum of possible virulence curves, producing a surface in three dimensions (right).

reproduce, it is not always in the best interests of a natural parasite to be as virulent as possible (Futuyma \& Slatkin, 1983; Maynard Smith, 1989; Combes, 1991; Hood, 1997). As a result, virulence varies dramatically between natural host-parasite systems (compare, for instance, cholera and the common cold), and over time within a particular system (e.g., the history of the myxoma virus in Australian rabbit populations, Fenner \& Ratcliffe, 1965). However, when parasites are used in artificial coevolution, they are generally encoded to be maximally virulent-their fitness varies inversely with the success of the hosts that they compete against.

The virulence of natural parasites strongly affects the coupling between host and parasite populations. Extremely virulent parasites may ultimately push their hostsand consequently themselves - to extinction, resulting in a decoupling of the system. As persistent high virulence can result in the disengagement of natural host-parasite systems, we enquire as to whether reduced parasite virulence could reduce the effects of disengagement in artificial coevolutionary systems-might coevolutionary algorithms benefit from treating parasites more naturally?

\subsection{Reducing Virulence}

Canonically, parasites receive fitness proportional to their ability to defeat the hosts they compete against. In order to reduce parasite virulence it is necessary to change this relationship. Throughout this paper we use the term score to refer to the ability of a parasite to defeat the hosts it is pitted against. Parasite scores are normalised with respect to the maximum score achieved that generation such that the best current parasite always achieves a score of 1 . We define parasite fitness as a function of score, $x$, and virulence, $\lambda(0.5 \leq \lambda \leq 1.0)$, such that:

$$
f(x, \lambda)=\frac{2 x}{\lambda}-\frac{x^{2}}{\lambda^{2}}
$$

Thus, a parasite achieves optimum fitness by winning a proportion of contests equal to a fraction $\lambda$ of that achieved by the best parasite. By varying $\lambda$, parasites can be encouraged to be more, or less, virulent (see figure 2). Although there is a continuum of possible curves, throughout this paper, we use only three values of $\lambda$. These are labelled as Maximum virulence ( $\lambda=1.0$, the equivalent of canonical parasites) where parasites are encouraged to beat as many hosts as possible, Moderate virulence $(\lambda=0.75)$ where 
parasites are encouraged to achieve a win-rate three-quarters that of the highest scoring current parasite, and Null virulence $(\lambda=0.5)$, where the fittest parasites achieve half the win-rate of their highest-scoring conspecifics. Notice that a value of lambda lower than 0.5 would always encourage cooperation between parasites and hosts, as they strive to achieve more host wins than losses.

It is important to emphasise at this point that drawing inspiration from natural systems does not make our approach more theoretically motivated than others, nor does it imply that biological systems necessarily optimise. However, we find it useful to visualise coevolutionary coupling in terms of virulence. As an alternative analogy, reducing virulence can be thought of as maintaining a gradient for selection, forcing parasites to evolve in difficulty at the same speed as the hosts by directly addressing the inherently asymmetrical advantage they possess. For example, in order to maximise pupils' learning, teachers must teach material that is neither too difficult nor too easy. Rather, teaching material should be presented on a smooth, continuous gradient of difficulty, consistent with the current academic requirements of pupils (e.g., Sklar \& Pollack, 2000).

\subsection{Experiment in a Minimal Domain: Counting Ones}

In order to introduce the concept of coevolutionary disengagement, Watson and Pollack (2001) used a minimal substrate to highlight its effects in the easily understandable Counting Ones domain. In this section we utilise an adaptation of the Counting Ones domain to demonstrate the effect that reducing virulence has upon coevolutionary disengagement when there exists an asymmetrical advantage favouring one population.

As described above, it is often the case that one side of a coevolutionary contest has an (at least temporary) advantage over the other in terms of the ease with which successful counter-adaptations are discovered. In complex coevolutionary systems, asymmetrical advantage is free to both fluctuate in amplitude, and even shift between populations. However, in order to aid analysis, the asymmetry introduced into the Counting Ones domain is fixed throughout each run. Although fixed asymmetry may not be representative of coevolutionary asymmetry in general, it allows us greater control whilst behaving in a manner similar (for our purpose, at least) to more realistic asymmetry.

Two reproductively isolated populations of size 25 are coevolved. Individuals in each population consist of binary strings containing $100 \mathrm{bits}$, with each bit initialised to 0 in generation 0 . The aim of the Counting Ones problem is to evolve strings containing as many ones as possible. Of course, in this toy example, as observers we can assess the absolute fitness or objective quality of each individual by counting its 1-alleles. This allows us, as experimenters, a useful way of measuring progress. However, the coevolutionary algorithm does not make use of this absolute measure, only having access to the relative fitness measure described below.

Members of one population are selected to play a set of pair-wise contests against a random sample of 5 opponents from the competing population. During each contest, the individual with the genotype containing the greatest number of 1-alleles receives a fitness point. Each opponent receives half a fitness point for contests resulting in a draw. Individuals in both populations reproduce asexually with parents chosen through tournament selection (tournament size 5; winner reproduces). Offspring have a small probability of mutation, $m$. Unless specified otherwise, the probability of mutation at each locus, $m$, was 0.03 .

An asymmetry was introduced by varying mutation bias $B_{p a r}\left(0 \leq B_{p a r} \leq 1\right)$ in favor of one of the two coevolving populations, henceforth classified as the parasite 

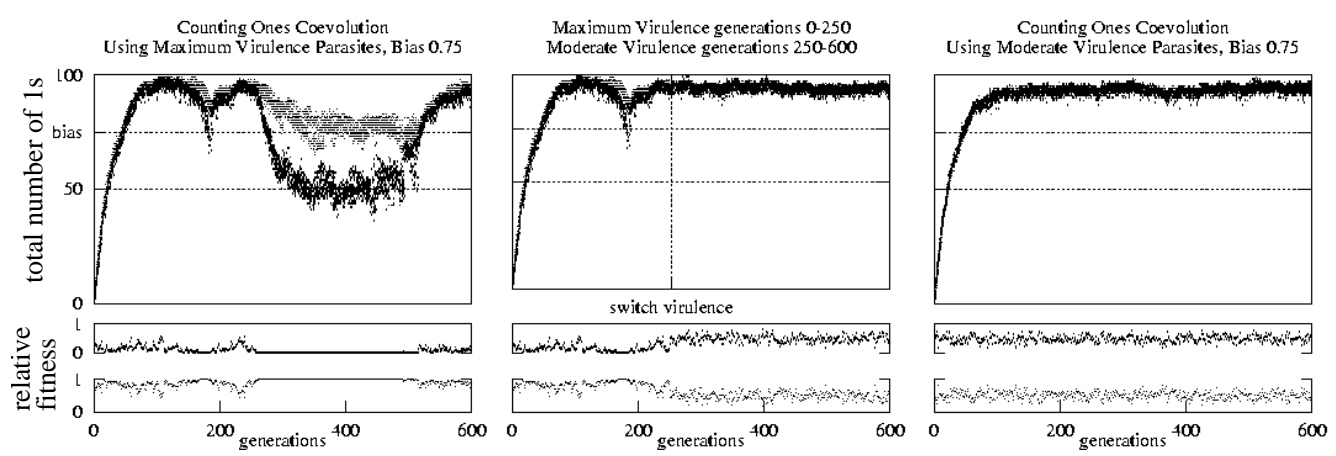

Figure 3: Results of typical coevolutionary runs in the Counting Ones domain with parasite mutation bias 0.75 , using the same random seed to initialise each run. With Maximum virulence parasites (left) there are two periods of disengagement. The second period may be prevented by switching to Moderate virulence at generation 250 (center). Populations remain engaged throughout the entire run when Moderate virulence is utilised from the beginning (right).

population. Given mutation at a particular parasite locus, the substitution of a 1 or 0 occurs with probability $B_{p a r}$ and $1-B_{\text {par }}$, respectively. In contrast, the coevolving host population substitutes a 0 or 1 with equal likelihood whenever mutation occurs. We thus see that if $B_{p a r}>0.5$, there is a bias in favour of evolving parasites with more ones-an asymmetry that favours the parasite population.

Two parasite mutation bias values and two parasite virulence levels were tested over a series of runs; $B_{p a r}=0.75,0.9$ and $\lambda=0.75,1.0$. Unless otherwise stated, the value of $\lambda$ remained constant throughout each run.

\subsection{Results}

Figures 3 and 4 each display three typical runs, using a parasite mutation bias, $B_{\text {par }}$, of 0.75 and 0.9 respectively. When employing maximally virulent parasites (figure 3 , left) the populations have a tendency to disengage. This can be observed between generations $150-175$ and again between $250-500$. During these periods of disengagement the populations drift back to their relative baseline performance, equal to the mutation bias, $B_{\text {par }}=0.75$ and $B_{\text {host }}=0.5$. Only once the populations re-engage by chance is there an improvement in absolute fitness. Repeating the run with the same random seed, the second period of disengagement depicted in figure 3 is prevented if Moderate virulence is introduced at generation 250 (figure 3, center). Notice that the left and center graphs are identical until generation 250-the point at which parasite virulence is changed to Moderate. In contrast to Maximum virulence, when Moderate parasites are used from the start (figure 3, right), the populations remain engaged throughout the entire run, achieving a continuously high level of performance.

With a parasite mutation bias of 0.9 , the increased asymmetry exacerbates the effects of reducing virulence. Typical of all runs, figure 4 , left, shows that a bias of 0.9 is too great for the host population to remain engaged with maximally virulent parasites after the initial 50 generations. However, runs employing Moderate virulence maintain population engagement despite the underlying asymmetry (figure 4, right). It should be noted, however, that whilst Moderate virulence helps to prevent disengagement from occurring, it does not encourage populations to re-engage. Switching to Moder- 

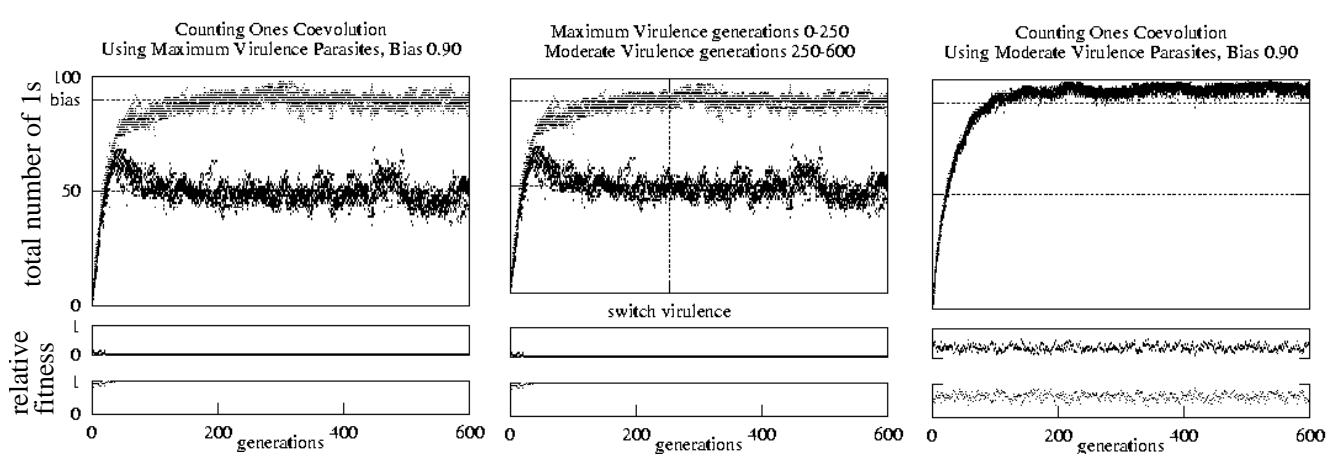

Figure 4: Results of typical coevolutionary runs in the Counting Ones domain with parasite mutation bias 0.9 , using the same random seed to initialise each run. With Maximum virulence parasites (left) the populations disengage within 50 generations and fail to re-engage. Switching to Moderate virulence during disengagement (center) has no effect. Utilising Moderate virulence from the beginning of a run (i.e., before the occurrence of disengagement) enables the populations to remain engaged throughout the run (right).

ate virulence during coevolutionary disengagement has no affect (figure 4 , center; see also section 4.3).

These results are sensitive to variation in both population size and the number of opponents played by each individual. As either parameter increases, the probability of disengagement decreases due to the increased frequency of meeting varied opponents. However, there remains a chance of disengagement even when sample and population sizes become very large - the phenomenon does not disappear. The results observed in this section are qualitatively robust to mutation rate $(m=[0.005,0.05])$ and tournament size (tourney $=[2,15])$.

\subsection{Discussion}

The results displayed in figures 3 and 4 clearly demonstrate that reducing parasite virulence in asymmetric coevolution can reduce the effects of disengagement. In particular, the greater the inherent asymmetry, the greater the effect reducing virulence has upon results. The asymmetry imposed in this model gave the biased parasite population a great advantage over the coevolving host population. Purely by stochastic effects one would expect individuals from parasite populations to contain more ones than those from host populations. This is observed in figures 3 and 4 . The difference in the speed with which the two populations initially move through the genotype space (resulting from the different mutation biases) ensures that disengagement occurs rapidly, and once it has occurred the same mutation biases tend to restrict each population to a different portion of the genotype space. Mutation bias pushes each population towards a particular ratio of ones to zeros, i.e., 0.5 for hosts and, dependent upon mutation bias, 0.75 or 0.9 for the parasites. As a result, both populations will remain disengaged until the gap between them is, at least temporarily, bridged by the occurrence of a very large number of mutation events, e.g., figure 3, left, generation 500 .

The first generation of parasite off-spring will on average contain many more ones than that of the host population. However, under reduced virulence, any parasite that beats all opponents is less fit than those parasites that lose a small percentage of con- 
tests. In this way, acceleration is decreased as the parasites resist their mutation bias. Moderate virulence parasites appear to actively prevent disengagement. Using the continued selection pressure ensured through engagement, hosts evolve to a higher objective quality than would otherwise be possible. It should not be overlooked, however, that Moderate parasites gain from this relationship too, as both populations evolve to a greater standard than either would alone (figure 4, right). However, as parasite virulence is decreased there is a tendency for coevolution to stagnate at a sub-optimal but highly engaged fluid local optimum. In order to push populations to optimal solutions, stronger selection pressure is required (see section 4.2, below).

\subsection{Defining Disengagement}

Full disengagement occurs when selection cannot distinguish between individuals irrespective of which sample of the current opponent population each individual plays. When assessment is noisy, although individuals in disengaged populations will achieve different scores, selection will not be able to distinguish between them on any systematic (i.e., non-random) grounds. In this situation, a coevolutionary algorithm has no basis upon which to preferentially select certain genomes for reproduction. Coevolutionary drift ensues, allowing deleterious mutations to accumulate in each population. Typically, in the course of running a coevolutionary algorithm, episodes of full disengagement will be difficult to distinguish from a much weaker kind of "contingent" disengagement. In this case, although each member of a population achieves identical, or near-identical fitness scores, they could have achieved different scores had they played alternative members of the current opponent population. We will use the term disengagement to refer to the complete lack of selective discrimination that results. The degree of disengagement, measured by how far (in terms of novel mutations) the populations are away from re-coupling, allows us to determine the likelihood of a reengagement event.

Asymmetrical disengagement occurs when one population, $P_{1}$, reaches the global optimum whilst the other, $P_{2}$, drifts through sub-optimal space, resulting in asymmetric selection pressure-whilst $P_{2}$ drifts, sub-optimal mutants from $P_{1}$ are selectively punished. An example of asymmetrical disengagement can be observed in the coevolutionary optimisation of sorting networks and test-lists. Once the sorting networks reach optimality, the selection pressure upon test-lists disappears-as all lists are sortable, each is equivalent. However, the sorting networks are under pressure to remain at optimality as sub-optimal mutants may be punished by test-lists ${ }^{2}$.

As an alternative analogy, asymmetrical disengagement may be observed in the coevolution of poker players. Players freely giving away information to opponentsvia either verbal or physical signals_-are disadvantaged. As opponents gain from correctly interpreting the signals that they receive, the optimal strategy for a player is to not signal at all. However, once a player stops signalling, all receiver strategies become equally redundant-there is no benefit in attempting to read any cues. Asymmetrical disengagement occurs when players reach the non-signalling optimum. Whilst receivers drift, any signaller deviation-i.e., starting to signal-may be punished.

\footnotetext{
${ }^{2}$ Asymmetrical disengagement may rapidly become full disengagement. In the case of the sorting networks, the drifting lists will likely become easier to sort in some absolute sense, reducing the pressure upon networks to remain at optimality.
} 


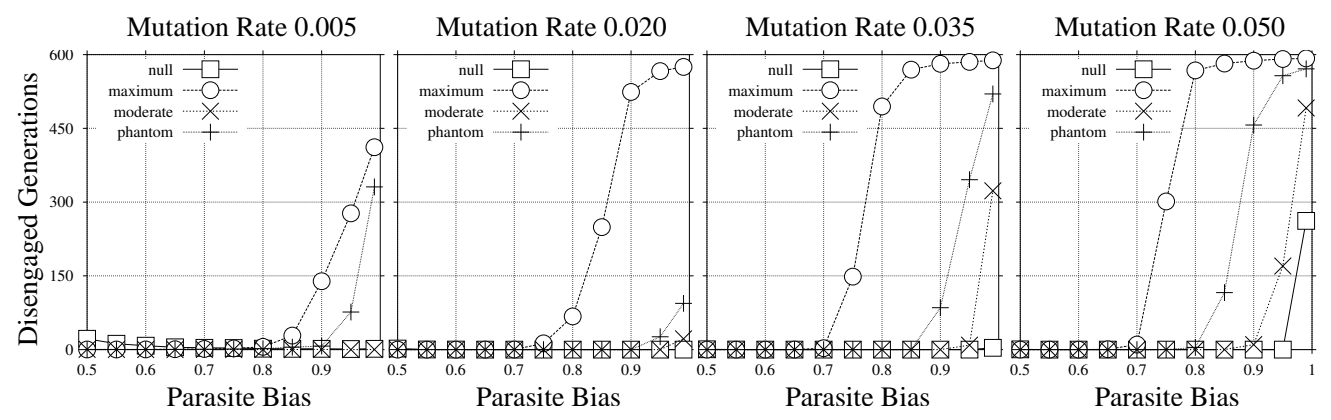

Figure 5: The average of 50 runs (each 600 generations) of Counting Ones coevolution, with mutation rate ranging between $m=0.005$ (far left) and $m=0.050$ (far right).

\section{Reduced Virulence: Further Experiments in the Counting Ones Domain}

In the previous section, we introduced a novel technique for maintaining engagement before demonstrating its potential in the Counting Ones domain. In this section we pursue further investigation in order to uncover exactly when reduced virulence is beneficial, how the value of $\lambda$ should be chosen and how a reduction in virulence compares to alternative engagement-maintenance algorithms. Throughout this section, unless otherwise stated, we use a mutation bias for parasites $B_{\text {par }}=0.75$, and per locus mutation rate $m=0.03$. Once again, the sample size of opponents is 5 and the tournament size for selection is 5 (winner always chosen to reproduce).

\subsection{Comparison with Phantom Parasite}

Of the engagement-maintenance techniques discussed in section 2.3, the only domainindependent algorithm versatile enough to fit the two-population coevolutionary model used for the Counting Ones domain is the Phantom Parasite (Rosin, 1997; figure 1, left). For this reason, we decided to compare reduced virulence with the Phantom Parasite, observing the behaviour of both algorithms when subject to varying levels of asymmetry and mutation. Maximum virulence $(\lambda=1.0)$, Moderate virulence $(\lambda=0.75)$, Null virulence $(\lambda=0.5)$ and the Phantom Parasite were each tested over a series of 50 runs with parameter values $B_{\text {par }}=[0.5,0.99]$ and $m=[0.005,0.050]$. Figure 5 displays the mean number of disengaged generations occurring each run.

Each graph clearly demonstrates the relationship between asymmetry and disengagement; irrespective of the engagement-maintenance technique, greater asymmetry (parasite bias) produces more disengagement. In contrast, disengagement occurs less frequently as virulence is reduced. Thus, reducing virulence is particularly beneficial when asymmetry is high. As the mutation rate increases, the effects of asymmetry are exacerbated. Given that the inherent asymmetrical advantage in favour of parasites is a mutational bias, any increase in mutation rate directly increases asymmetry.

As we would expect, the Phantom Parasite performs better than canonical parasites $(\lambda=1.0)$ across all levels of asymmetry and mutation. However, it can be observed that the Phantom Parasite is sensitive to mutation rate-rather than exhibiting a monotonic relationship between disengagement and mutation rate, disengagement increases as $m$ diverges from 0.020 . The Phantom Parasite thus behaves less predictably than reduced virulence, perhaps due to the discontinuity in the gradient of the fitness curve (see figure 1, left). In contrast, the Phantom Parasite is not as effective as Moderate or 
Null virulence in reducing disengagement when asymmetry is high. Again, this is as we would expect. The Phantom Parasite is most like reduced virulence with $\lambda=\frac{N-1}{N}$ (thus sensitive to sample size $N$, here $N=5$ ), with the exception that it is always best to win all competitions. As such, the Phantom Parasite, here similar to $\lambda=0.80$, performs better than Maximum but worse than Moderate and Null virulence.

Thus, in comparison with the Phantom Parasite, reduced virulence acts more predictably (is less sensitive to coevolutionary parameters), produces less disengagement (assuming $\lambda$ has been chosen adequately) and is more flexible (i.e., $\lambda$ can be varied as required).

\subsection{Trade-off: Engagedness versus Optimality}

The reduced virulence technique has been observed to limit the effects of disengagement, however, the effect upon performance (in this case, the total number of 1-alleles within the genome of the best host) has not been considered-as reducing virulence directly interferes with the selection pressure upon parasites, it is likely that the performance of the coevolutionary system will be affected in some way.

Figure 3 hints at an answer. When utilising Maximum virulence parasites (figure 3, left), the system briefly reaches near optimal performance (generations 100-150) before disengaging. However, when using Moderate virulence parasites (center and right), maximum performance is sub-optimal, despite being much more stable. As Maximum virulence parasites result in the strongest selection pressure upon hosts, we hypothesise that-given that a coevolutionary system can maintain engagement for a sufficient period of time-Maximum virulence will push a system to a greater level of performance than that achieved by parasites with reduced virulence.

This hypothesis was tested by forcing populations to remain engaged throughout a run. In a variant of the counting ones experiment, we assessed each member of a single population against a random sample of opponents drawn from the same population. In this way, to the extent that the population remains phenotypically diverse, it must remain engaged with itself over evolutionary time.

For each virulence level, 30 runs were performed. After approximately 100 generations the coevolutionary system settled into an equilibrium at its highest level of performance. With a $95 \%$ confidence limit, the equilibrium level of performance for each virulence level was: Maximum $96 \pm 2 \%$, Moderate $89 \pm 2 \%$ and Null $75 \pm 2 \%$. It appears that, if engagement can be guaranteed, maximum performance increases with virulence.

Thus, performance is maximised by setting virulence to be as great as possible, without sacrificing engagement. The trade-off between engagement and performance must be balanced by choosing the optimal level of virulence, $\lambda$. However, once a system has disengaged, it is not obvious which virulence level is optimal. This is discussed in the following section.

\subsection{Encouraging Re-engagement}

The results in section 4.1 show that reducing virulence can limit the propensity for asymmetric populations to disengage.

However, as yet we have gained no insight into the effects that parasite virulence has upon populations that are already disengaged. Is it possible that certain virulence functions increase population diversity and so encourage populations to re-engage? In order to test this hypothesis, we experimented by forcing population disengagement at the start of each run. This was achieved by initialising every individual in the host 

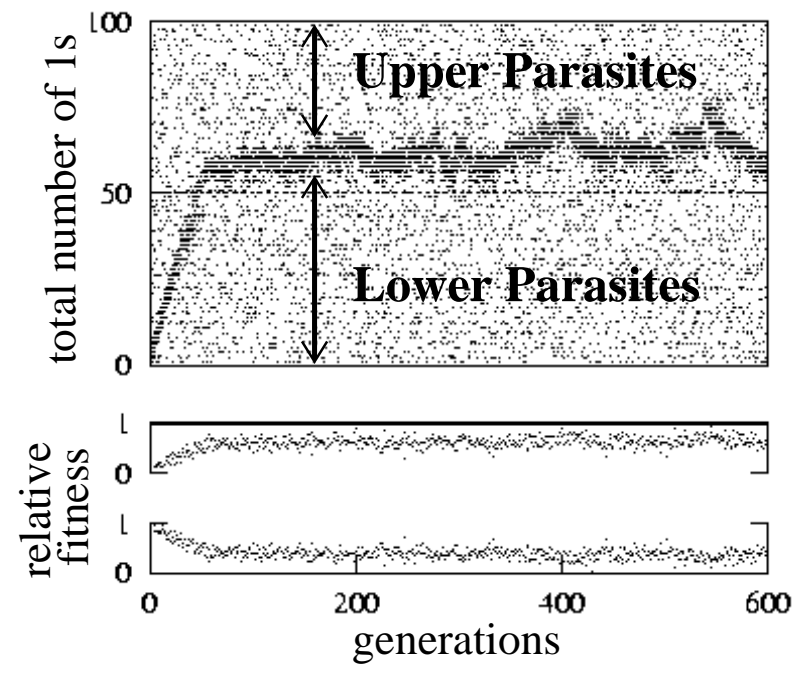

Figure 6: Random parasites with no heredity. Although most parasites are either "upper" or "lower" parasites, the likelihood is that some parasites will fall within the set of strategies capable of discriminating between hosts. This produces a small selection pressure encouraging the objective quality of the host population to fluctuate around $10 \%$ higher than that achieved by mutation bias alone.

population to a genotype consisting of zeros, whilst initialising individuals in the parasite population to have some integer, $i>0$, number of ones in their genomes. In each run, the number of generations until first engagement was recorded across a range of values of $i$. Using the same initial conditions (i.e., same random seed), 30 runs were performed using Maximum, Moderate and Null virulence parasites. Results demonstrated absolutely no difference in the time to re-engagement-the time until populations first engage is independent of virulence: it is purely a stochastic process. The host and parasite populations drift until an instance arises when at least one parasite does not score maximally against at least one host. This is a re-engagement event.

Although the time to first engagement is independent of parasite virulence, the behaviour of the system henceforth most certainly is not. Imagine a situation where every parasite but one scores maximally and every host but one scores minimally. With a Moderate or Null virulence scheme the non-maximal scoring parasite would be rewarded with greater reproductive success. The same would be true of the non-minimal scoring host. Effectively, the populations would be drawn together and engagement would be encouraged. With a Maximum virulence scheme, however, the situation would be very different. Rather than be rewarded with progeny, the non-maximum scoring parasite would be unlikely to have any progeny at all whilst every other parasite would produce marginally more offspring than before. Effectively, we would see the populations bounce apart-disengagement is likely to re-occur the very next generation.

\subsection{Random Parasites: Canonical Evolution}

In this section, we consider how coevolution compares to canonical evolution in the Counting Ones domain. Canonical evolution is implemented by evolving a population 
of solutions (hosts) against non-hereditary test-problems (parasites), randomly selected at the beginning of each generation with a uniform distribution of densities (total number of $1 \mathrm{~s}$ in the genome).

Figure 6 displays the output of one typical run. As can be seen, the host population fluctuates in performance around $60 \%$-alleles-10\% higher than the population would tend to by mutation alone-implying random parasites are exerting some selection pressure. Let us consider those parasites that fall above the host population-the upper parasites. By definition, each competition between a host and upper parasite will result in victory for the parasite. Conversely, consider those parasites falling below the host population-the lower parasites. In this case the reverse will be true. Each competition will result in a win for the host. Thus, how strong or weak a host is in relation to other hosts cannot be discerned on the basis of competitions with either upper or lower parasites. The host population is disengaged from the upper and lower parasites.

It is likely, however, that some parasites will engage with the host populationdiscriminating parasites. These individuals are able to discern, to some degree, the relative strength of hosts, thus producing systematic selection pressure. However, due to the relatively tight distribution of hosts-in terms of possible distributions, the host population is relatively converged-only a small proportion of parasites are able to discriminate. As such, Random non-hereditary parasites exert a constant, but relatively weak, selection pressure-the system has a small degree of engagement.

The impetus behind coevolving test-problems with solutions derives from the desire to automatically sample tests at the required difficulty, reducing the necessary number of tests and resulting in computational efficiency. The effectiveness of coevolution in improving performance can be observed by comparing figure 3, right, and figure 6coevolving hosts fluctuate around $30 \%$ higher than those evolving with random parasites.

However, the occurrence of disengagement drastically changes this relationship. Disengaged populations experience no selection pressure and thus drift to their respective base-line levels of performance. Fluctuating at around 50\% 1-alleles, disengaged hosts perform worse than hosts assessed through standard evolution. However wasteful random parasite populations are in terms of discriminatory ability per parasite, the small but continuous selection pressure they exert is better than long periods with no selection pressure at all. Although coevolution may have the ability to outperform standard evolution in certain domains, long episodes of disengagement can easily reverse this advantage.

\subsection{Conclusions from the Counting Ones Domain}

Several conclusions can be drawn from the experiments in the Counting Ones domain, however, we cannot assume that these are true in general. Firstly, there exists a tradeoff between reducing virulence to encourage engagement and increasing virulence to improve performance. Secondly, disengagement can reduce the performance of coevolutionary algorithms to below that of standard evolution, even if the domain is particularly suited to a coevolutionary approach. Once disengagement has occurred, the level of virulence is irrelevant-re-engagement occurs stochastically. Nevertheless, reducing virulence increases the likelihood of prolonging re-engagement once it occurs. Finally, reduced virulence outperforms its closest competitor, the Phantom Parasite.

Evolutionary Computation Volume x, Number $\mathrm{x}$ 

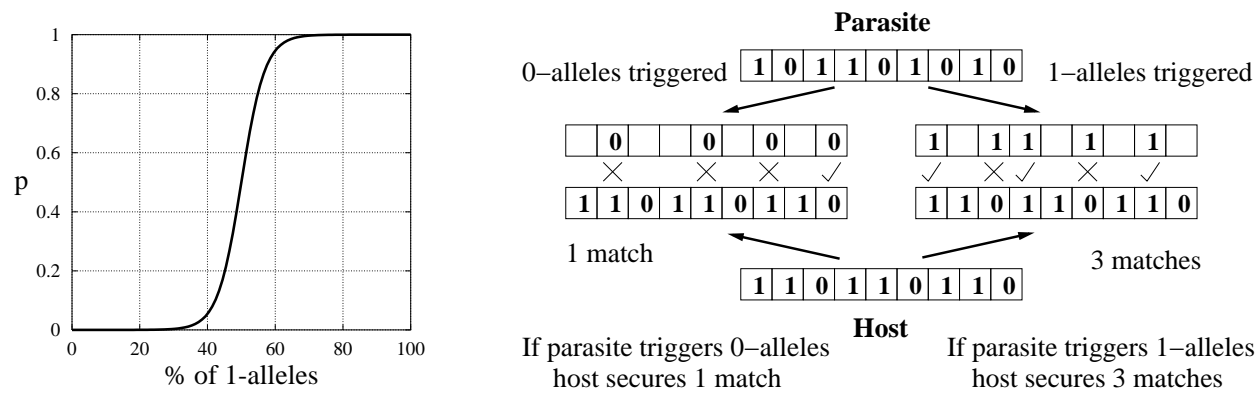

Figure 7: The probability, $p$, of parasites playing the 1-allele half of the matching game (left, see also equation 3). Depending upon which half of the matching game the parasite plays, hosts try to match either 0 - or 1-alleles (right). If matches $\geq T$ then the host wins, otherwise the parasite wins.

\section{Reduced Virulence versus Diversity Maintenance}

Disengagement occurs when intra-population fitness diversity reduces to zero. Moderating virulence counter-acts disengagement by selecting for reproduction parasites that are occasionally beaten. This preserves a selection gradient for hosts which, in turn, maintains relative fitness diversity in both populations.

A tendency towards reduced population diversity (and the associated problem of premature convergence) has long been a major concern of the evolutionary computation research community. As such, a suite of diversity maintenance techniques have been proposed, including e.g., deterministic crowding (De Jong, 1975), explicit fitness sharing (Goldberg \& Richardson, 1987), competitive fitness sharing (Rosin, 1997; Rosin \& Belew, 1997), resource sharing (Juillé \& Pollack, 1998b), and spatial embedding (e.g., Hillis, 1990). These approaches are attempts to maintain genetic diversity on the assumption that a loss of diversity can be harmful to optimisation as it may restrict search to local optima.

Resource (or competitive fitness) sharing maintains genetic diversity in a population by encouraging niching - individuals are rewarded for being able to solve tests that few others can. This idea has been extended to coevolutionary scenarios where opponents are treated as a commodity or resource. Rather than gain a fitness point for each victory against an opponent (simple fitness), one fitness point is shared among the competitors that beat a particular individual. Thus, individuals are rewarded less for how many opponents they beat and more for who they beat, rewarding phenotypic diversity and maintaining genetic diversity.

Since disengagement is associated with a loss of diversity, could it be prevented by simple diversity maintenance approaches? Perhaps reducing virulence is only preventing disengagement by mimicking these existing techniques? If so, it is largely superfluous. In the next study we contrast reduced virulence with resource sharing in order to explore whether they are effectively the same or different in some fundamental sense.

\subsection{The Matching Game}

In order to compare the influence of parasite (and host) resource sharing with that of reduced parasite virulence, we need to choose an appropriate and simple problem domain. Here we develop a simple matching game, in which hosts are rewarded for matching parasites, but parasites are punished. Games with this type of dynamic of- 
ten suffer from coevolutionary cycling, as hosts chase parasites through the strategy space. Although desirable generalist strategies exist, populations are easily diverted from them as they exploit the temporary idiosyncrasies of their opponents. Resource sharing is one way of discouraging this type of short-termist behaviour. By maintaining a diverse strategy-base in each population, the value of exploiting idiosyncrasies is reduced, encouraging generalists. Unfortunately, an alternative mediocre stable scenario is possible in which populations "speciate" such that they exhibit a number of different sub-optimal strategies that together form a stable combination. In this sense, the game is similar to any number of scenarios in which a generalist strategy is desirable (from the perspective of the optimiser), but difficult to evolve in practise-e.g., scissors-paper-stone, immune systems, etc.

Two distinct populations of size 50 are coevolved-hosts and parasites. Individuals in each population consist of binary strings containing 100 bits, initialised randomly in generation 0 . Each generation, members of the host population are selected to play a set of pair-wise contests against a random sample of 10 opponents from the parasite population. The aim for hosts is to match as many parasite alleles as possible. Antagonistically, parasites aim to mis-match host alleles. Both populations breed asexually, with each individual having a small probability of unbiased mutation per locus, $m=0.03$. Tournament selection was used (tournament size 5) with the winner of each tournament always chosen to reproduce.

Not all loci are involved in this matching game. For parasites with many 1-alleles, the matching game tends to involve only those loci at which the parasite possesses 1alleles. For parasites with many 0 -alleles, the game tends to involve only those loci at which the parasite possesses 0 -alleles. Whether 1-allele loci or 0-allele loci are involved is determined probabilistically. The probability, $p$, of a game involving matching 1alleles increases with the total number of 1 -alleles, $x$, such that

$$
p=\frac{1}{2}\left\{1+\tanh \left(\frac{x-50}{7}\right)\right\}
$$

Once the game has been decided, a host wins by matching alleles in at least $T=30$ loci, else the parasite wins (see figure 7).

Having several antagonistic points of attraction, the Matching Game domain is designed to exhibit interesting coevolutionary dynamics. Mutation bias attracts both populations towards genotypes containing $50 \% 1$-alleles and $50 \% 0$-alleles. However, given a host plays a parasite at the 1-allele (0-allele) half of the matching game, it is advantageous for the host to have as many 1s (or 0s) as possible. Thus, the host population is attracted towards homogeneous genotypes (all 1s or all 0s). The direction of attraction for hosts (towards either $100 \% 1$ - or 0 -alleles) depends upon the frequency with which the parasite population plays either the 1-allele or 0-allele halves of the game. This occurs with increasing frequency the further parasite genotypes vary from $50 \%$ 1s. Thus, parasites are also attracted away from $50 \% 1 \mathrm{~s}$, but in the opposite direction to hosts. Parasites deviating too far from $50 \% 1 \mathrm{~s}$, however, become too predictable. In general, the most difficult parasites to match are those having approximately $50 \% 1 \mathrm{~s}$.

This matching game resembles the density classification task for 1-D cellular automata, for which Juillé and Pollack (1998a, 1998b) and Pagie and Hogeweg (2000) utilised a method of virulence reduction-the $\Phi$ function (refer to section 2.3). The density classification task for cellular automata is difficult-no rule set exists which can correctly classify all ICs (Land \& Belew, 1995)—as such, consistently coevolving two populations towards continuous improvement is problematic (Paredis, 1997). Whilst 

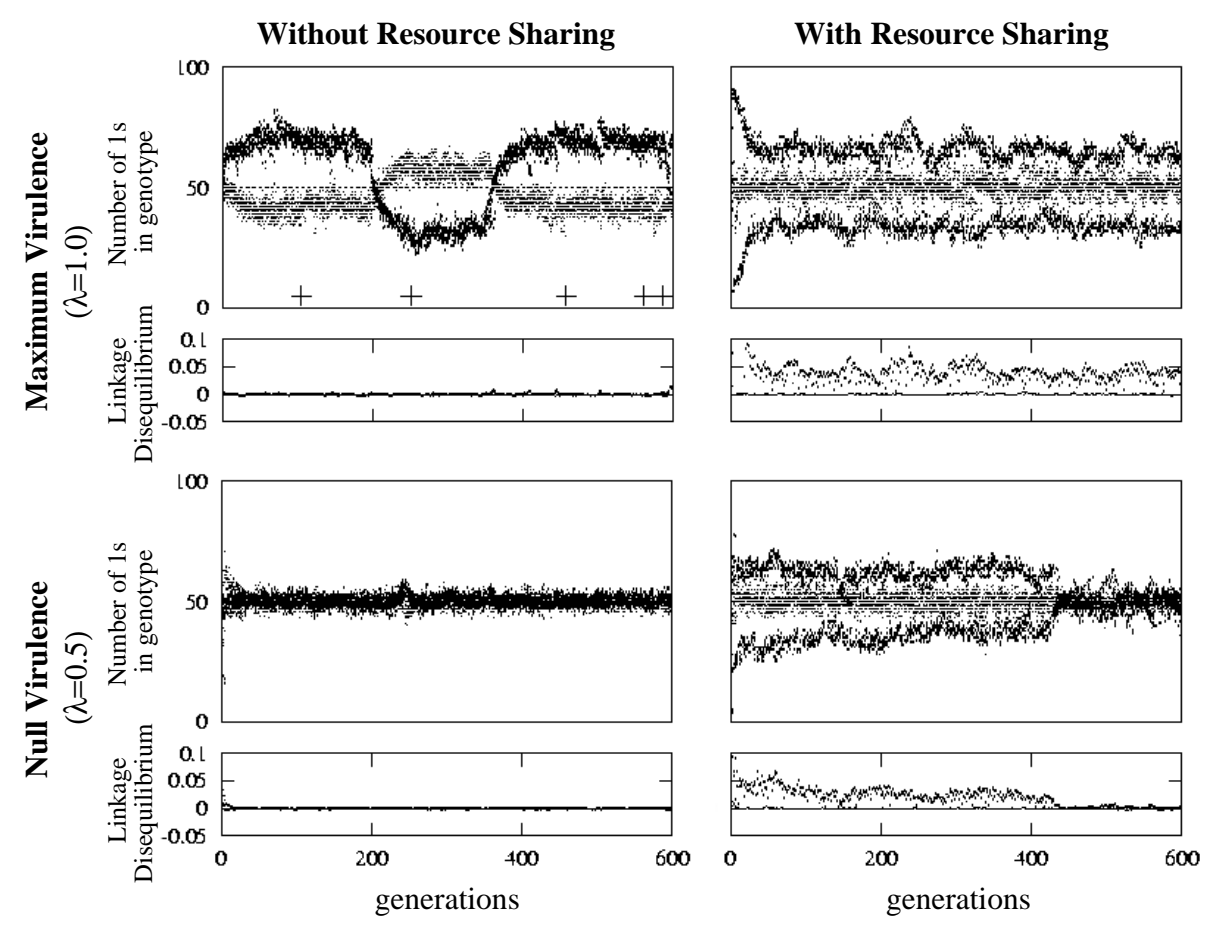

Figure 8: Typical coevolution in the Matching Game domain. Maximum virulence without resource sharing produces cycling as hosts repeatedly alternate strategy (topleft). Maximum virulence with resource sharing leads to mediocre stability with half the host population focusing upon each strategy (top-right). Null virulence without resource sharing encourages hosts to become generalists, capable of either strategy (bottom-left). Null virulence with resource sharing initially pushes hosts into two specialist niches, before funnelling the population into generalists (bottom-right).

coevolving CA rules, Juillé and Pollack found it necessary to "reduce the virulence" of ICs in order to stop disengagement, despite the use of resource sharing. Here we tease apart the contribution of two domain-general approaches to improving coevolutionary optimisation, resource sharing and reduced parasite virulence.

Two $\lambda$ values were tested over a series of runs; $\lambda=1.0$ (Maximum) and $\lambda=0.5$ (Null). The value of $\lambda$ remained constant throughout each run. Runs were performed under four conditions: Maximum virulence without resource sharing (i.e., standard coevolution); Maximum virulence with resource sharing; Null virulence without resource sharing; both Null virulence and resource sharing. Under each condition, the degree of niching or genotypic diversity within each population was calculated using a linkage disequilibrium measure (e.g., Barton \& Gale, 1993) particularly sensitive to the effects of resource sharing.

\subsection{Results}

Figure 8 displays four typical graphs from the Matching Game domain, resulting from the four test conditions. Both resource sharing and reduced parasite virulence have clear effects on coevolutionary dynamics.

Under condition one-Maximum virulence with no resource sharing, i.e., typical 
coevolutionary optimisation (figure 8, top-left)—the system exhibits cycling. After the initial generations, hosts may begin to recruit more 1-alleles in order to defeat parasites playing the 1-allele half of the game. However, as parasites counter-adapt, by recruiting more 0 -alleles, they increase the likelihood of playing the 0 -allele half of the game. In response, hosts appear with a greater proportion of $0 \mathrm{~s}$, with the entire population eventually switching strategy, in order to concentrate on winning the 0-allele half of the game. Subsequently, parasites again regain the upper-hand by recruiting 1-alleles, and so on. Under these conditions, the Matching Game is inherently easier for the parasite population. Hosts find it difficult to be successful generalists-incapable of matching parasites along both dimensions - and are encouraged to become brittle specialists. As a result, maximally virulent parasites win the majority of competitions and occasionally win all competitions, resulting in disengagement (indicated by crosses).

Under condition two-Maximum virulence with resource sharing (figure 8, topright) - the system reaches mediocre stability. At the beginning of the run, hosts immediately niche into two groups, each specialising on one half of the matching game. In order to be as unpredictable as possible, parasites tend towards $50 \% 1 \mathrm{~s}$-any deviation from this distribution will be punished by one of the specialist host niches. At this mediocre equilibrium the host population as a whole achieves roughly $50 \%$ victories over parasites, but each individual host is extremely vulnerable to parasites playing the opposite half of the game. In contrast, parasites tend to become maximally unpredictable and play either half of the matching game with roughly equal probability.

Under condition three-Null virulence $(\lambda=0.5)$ without resource sharing (figure 8 , bottom-left)-the system stabilises with generalist hosts. After the initial generations, the host population settles into generalist strategies, capable of matching some parasites whichever allele is triggered. Moderate virulence ensures that parasites are rewarded when occasionally matched, thus allowing hosts to succeed without having to concentrate on winning one half of the matching game. It should be noticed that reducing virulence does not result in host-parasite collusion, which would tend to result in homogeneous parasites-the simplest to match. Rather, parasites remain challenging and unpredictable. Any deviation from $50 \% 1 \mathrm{~s}$ is quickly punished by the generalist hosts. As such, both hosts and parasites engage in competition in the most difficult regions of space. This is equivalent to discovering the "play random" strategy in scissors-paper-stone, or a generalist immune system capable of defeating a wide range of intruders.

Under condition four-Null virulence $(\lambda=0.5)$ with resource sharing (figure 8 , bottom-right) - the system initially achieves mediocre stability, before encouraging hosts to become generalists, strongly engaged with parasites. Early in the run, resource sharing encourages the host population into two niches, each concentrating on one half of the matching game. In this way, the system reaches mediocre stability with hosts and parasites sharing victories. However, unlike condition two, mediocre stability does not persist. Recall that Null virulence encourages parasites to achieve a win-rate half that of the highest scoring parasite. This scheme lures parasites away from the mediocre equilibrium at which they achieve a $50 \%$ win-rate. As parasites become more easily matched, they reduce the pressure upon hosts to concentrate on one half of the matching game. In this way hosts are steered towards a more generalist strategy of $50 \% 1 \mathrm{~s}$. Hosts engage parasites in a difficult region of space, unattainable without a reduction in parasite virulence.

Results clearly demonstrate that imposing reduced virulence on parasites alters coevolutionary dynamics in a fundamentally different way to that achieved by resource 
sharing. Whilst resource sharing encourages within-population genetic (and phenotypic) diversity, observable as niching in the host population, reduced virulence encourages diversity in relative fitness (i.e., a between-population phenomenon).

\subsection{Caring versus Sharing}

Resource sharing encourages a population to diversify into separate niches, thus reducing the likelihood of over-focusing. In this way, coevolutionary cycling may also be avoided. However, niching may produce mediocre stability-or sub-optimal equilibrium-whereby niches share success. In contrast, reducing virulence does not encourage intra-population diversity and rather encourages engagement-the extent to which coevolving populations interact.

Resource sharing adds a second layer of coupling between conspecifics. In addition to the standard competition that conspecifics experience-striving to beat more opponents than each other-they are forced to share their success with one another. This encourages individuals to beat different opponents-i.e., to be different from one another. Niching results from this additional intra-population coupling.

In contrast, reducing parasite virulence increases inter-population coupling-it ensures that individuals in one population care about the success of individuals in the other. In particular, through attempting to achieve moderate success, parasites care about the variation in relative fitness achieved by their opponents-they are selected to cause a range of scores in their opponents. However, this is not achieved through niching, or genetic diversity per se. Rather, it is a direct consequence of the moderation that maintains engagement.

It is true that increased genetic diversity has some relationship with coevolutionary engagement. If genetic diversity reduces to zero, populations will disengage (individuals will achieve equivalent scores). However, the converse is not true. Genetic diversity does not ensure engagement. Both populations may feature a diverse array of phenotypes, yet still suffer disengagement if each and every phenotype in one population defeats each and every phenotype in the other. Indeed, periods of disengagement often increase genetic diversity through random drift without necessarily increasing engagement. While this coevolutionary coupling (engagedness) is affected by genetic diversity (and noise, sampling error, etc.), it is not determined by it.

These considerations ensure that reducing virulence and resource sharing are complementary, rather than exclusive, tools. It is not necessary to choose one over another. Indeed, the greatest success may result from using diversity and engagement maintenance techniques in conjunction (Juillé \& Pollack, 1998a, 1998b).

\section{Application in a Complex Domain}

Throughout our investigations in the simple Counting Ones and Matching domains, evidence has been gathered to support the hypothesis that reducing parasite virulence can encourage coevolutionary engagement. However, it is not obvious that these results generalise to other, more complex, domains. In order to address this issue, reduced parasite virulence is applied to the coevolution of minimal length sorting networks-a domain that has historically attracted interest from the coevolutionary computing community. The aim is to design the shortest fixed network of comparisons that can sort, into numerical order, any input list containing a specific number of elements. Comparisons exist in the form if $a>b$ then swap, else do nothing. 


\subsection{List Sorting Algorithms}

Hillis' (1990) seminal work proved by example how artificial coevolution can be applied to optimization problems. Hillis chose to design minimum comparison sorting networks, a domain with a long and competitive history that has resulted in the current record of sixty comparisons to sort sixteen elements, achieved by hand in 1960 by M. W. Green (Knuth, 1973). Initially, using randomly generated inputs as test sets, Hillis evolved the sorting networks on a static fitness landscape, resulting in a minimal network of 64 comparisons. He found that two factors were preventing the evolution of shorter networks. Firstly, the classical problem of local maxima made it difficult for the system to progress once a reasonable solution had been found. Secondly, the test process proved inefficient-after a few generations most of the inputs were fully sorted by the vast majority of networks. To compensate for these problems, Hillis allowed the test cases to coevolve with the sorting networks, giving them complimentary fitness functions and thus producing an artificial host-parasite system. This allowed the lists to evolve towards punishing the weaknesses of suboptimal networks, whilst dramatically reducing the number of redundant tests-those too easy to sort. Using this method, Hillis discovered a 61-comparison network, a much better solution than previously evolved and only one comparison longer than the best known solution.

In order to encourage population genetic diversity Hillis utilised spatial embedding. By evolving individuals upon a toroidal grid and allowing only local interactions, spatial embedding supports the formation of multiple niches within each population. As such, spatial embedding can be considered as an alternative approach to other niching techniques such as resource sharing or fitness sharing (Pagie \& Hogeweg, 2000). In conjunction with spatial embedding, Hillis used a very weak selection pressure-in each generation the bottom $50 \%$ of individuals were culled, leaving the top half to breed at random. Thus, to ensure maximum chance of reproduction, an individual need only be ranked among the top half of the population. Such weak selection upon parasites may encourage engagement in a similar fashion to reduced virulence. In combination, spatial embedding and culling may have had a profound affect upon Hillis' results.

Throughout the last decade, Hillis has inspired much research in the domain of coevolving sorting networks (e.g., Juillé, 1995; Olsson, 1996; Rosin, 1997). Most impressively, Juillé's Evolving Non-Determinism (END) model improved upon a 25-year-old record by discovering a minimal comparison network of length 45 for list inputs of 13 elements.

\subsection{Setup}

For historical reasons ${ }^{3}$ we have chosen to coevolve sorting networks for 13-element input lists. Rather than attempt an assault on the minimal length record, however, we aim to record the difference in performance that varying parasite virulence induces.

Our model is loosely based upon Hillis' original scheme, however, as Hillis was primarily interested in optimisation, several changes are implemented. Hillis utilised very large populations (of the order of $10^{6}$ individuals), sexual recombination, and seeded initial populations with the butterfly ${ }^{4}$ (Rosin, 1997). As our primary aim is not to find an optimal network, our model can be simplified by removing sexual recombination, reducing population sizes and initialising individuals at random (i.e., no butterfly seeding). In order to test the effect of spatial embedding, runs are performed under two conditions; with spatial embedding and without spatial embedding. Under both

\footnotetext{
${ }^{3}$ The shortest network currently known for this problem was discovered using a coevolutionary algorithm (Juillé, 1995).
}

Evolutionary Computation Volume $x$, Number $x$ 
conditions, hosts and parasites evolve on a toroidal grid, with exactly one host and one parasite occupying each location and playing each other.

With Spatial Embedding For each grid location, $g$, a tournament is played between individuals in the Von Neumann neighbourhood (i.e., center square and 4 nearest neighbours; North, East, South, West). The highest scoring host and parasite leave (perhaps mutated) progeny in the center square, $g$, the following generation.

Without Spatial Embedding For each grid location, a host and a parasite are chosen from two tournaments of 5 individuals each, randomly selected from across the gridhighest scoring individual always chosen—each leave (perhaps mutated) progeny at $g$.

Host networks consist of 45 pairs of integers, with each pair representing list elements to be compared and, if necessary, swapped. Host mutation occurs at each loci with probability 0.02 , producing a random integer in the range 1 to 13 . Parasites each contain 40 unsorted 13-element lists. In order to preserve lists as permutations of the integers 1 to 13, parasite mutation consists of swapping two elements of the list-this occurs at each loci with probability 0.02 . Both parasite and host population sizes are identical, with each host attempting to sort exactly one parasite- that which shares the same grid location. Without spatial embedding, therefore, parasites and hosts are essentially paired at random each generation, however, with spatial embedding, specific host and parasite lineages are more likely to meet repeatedly over multiple generations. Both hosts and parasites are asexual.

A host is rewarded with fitness proportional to the number of parasite lists that are completely sorted. Reciprocally, parasites are rewarded for possessing lists that remain unsorted. Maximum, Moderate and Null virulence and the Phantom parasite were each tested. In conjunction, Random parasites with no heredity were also tested so as to compare the coevolutionary results of each virulence scheme against what is effectively standard evolution. In order to collect accurate statistics, 30 runs were carried out for each condition with population sizes ranging from 25 to 225. An advantage of this problem domain is that an absolute, objective fitness measure of hosts is possiblenetworks are given every possible input to sort ${ }^{5}$ with the percentage of correctly sorted lists determining an absolute fitness performance. A host network that can sort $100 \%$ of all possible inputs is an optimal network.

Under each condition, performance is compared by calculating the mean of the absolute performance of the best individual discovered each run. It should be noted that the coevolutionary system has no knowledge of this absolute fitness performance, it is merely a way to record comparable results.

\subsection{Results}

Figure 9 displays the results of coevolving sorting networks both with and without spatial embedding, under the 5 conditions labelled, Maximum, Moderate, Null, Phantom and Random. Each graph shows the mean (over 30 runs) of the current best-so-far network performance.

\footnotetext{
${ }^{4}$ The first 32 exchanges (the butterfly) in Green's 60-comparison network are known to sort the vast majority of inputs. As such, Hillis seeded every initial individual with the butterfly in order to encourage optimisation.

${ }^{5}$ The set of input lists can be calculated efficiently by using the zero-one principle: 'a network can sort every input list if and only if it can sort every binary input list' (Knuth, 1973).
} 


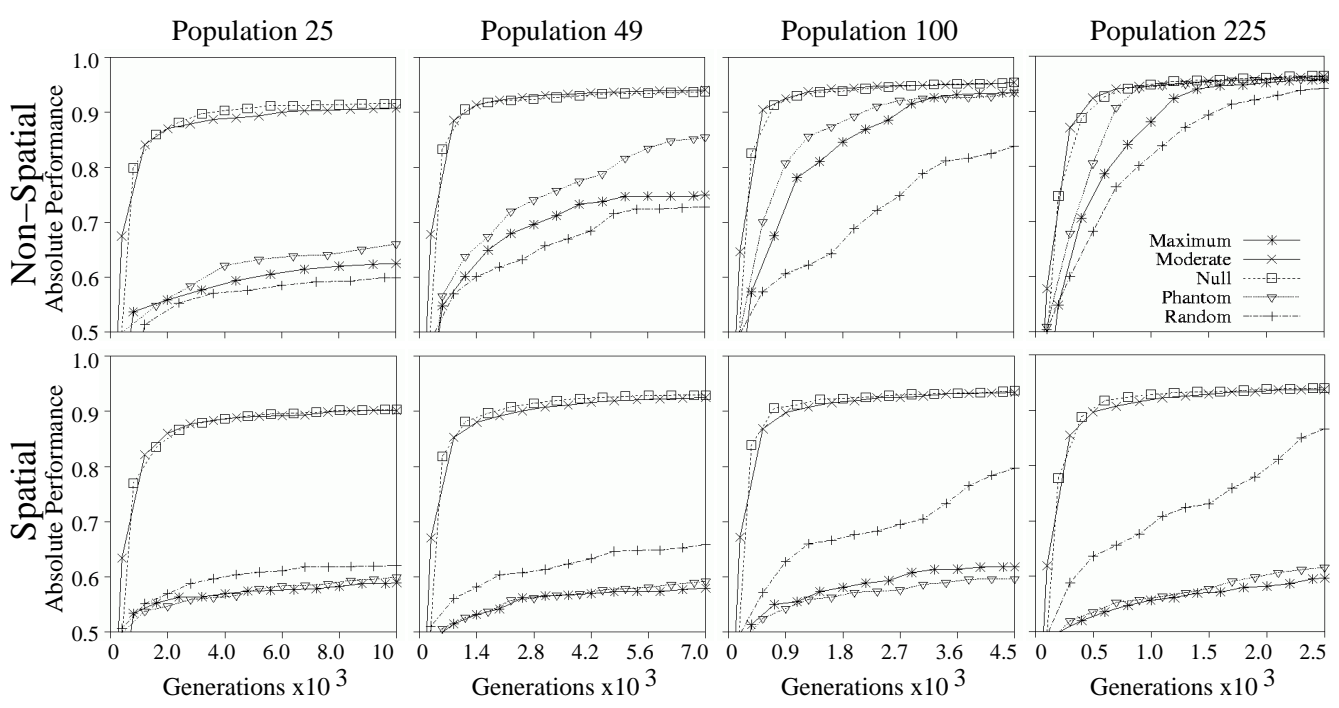

Figure 9: Coevolution of minimal length sorting networks. As population size increases, the number of generations graphed is reduced in order to depict approximately equivalent lengths of units of computational time.

Without spatial embedding (figure 9, top), the Moderate and Null conditions significantly outperform Maximum, Phantom and Random conditions, particularly when population size is small. However, this performance difference reduces as population size is increased, suggesting that-since disengagement becomes progressively less likely as population sizes increase-reducing virulence improves performance by diminishing the effects of disengagement (rather than for some other reason).

Maximum does not perform significantly better than Random until population size reaches 100 . Considering the weak selective pressure induced by Random parasites this is a poor result, again suggesting that Maximum virulence produces long periods of disengagement. Indeed, upon scrutinising individual runs (not shown), this can be observed.

When spatial embedding is implemented (figure 9, bottom), Moderate and Null conditions significantly outperform Maximum, Phantom and Random conditions across all population sizes. Once again, this can be understood in terms of disengagement. Spatial embedding exacerbates the asymmetrical advantage favouring parasites-as host and parasite lineages repeatedly meet over many generations, parasites are able to specialise against specific host weaknesses (over-fitting or overfocusing). As spatial embedding allows only geographically local interactions, this effect is largely independent of population size. Whilst the Maximum condition ensures that it is very difficult for hosts to engage, Moderate and Null encourage engagement by stopping parasites from over-fitting host idiosyncrasies, thus resulting in improved performance.

These results are suggestive, but are certainly not conclusive. Evolving minimumcomparison sorting networks is a complex problem domain, making it difficult to analyse. However, we would expect the list-sorting problem to exhibit the kind of asymmetry that led to disengagement in the counting-ones problem. Being a challenging set of lists is much easier, at least at the outset of coevolution, than being an accomplished list 
sorter. Could this asymmetry account for the relatively slow progress made by conventional coevolution? If this type of initial asymmetry is a feature of many coevolutionary optimization problems, reducing parasite virulence in some way could turn out to be an approach with wide application.

\subsection{Discussion: Neutrality}

Throughout the paper we have described how coevolutionary disengagement results in periods of evolutionary drift. Recently, within the evolutionary computation community, there has been some interest in the role of neutral drift in the behaviour of evolutionary algorithms (Barnett, 1998). It has been suggested that neutral drift might alleviate problems of premature convergence, and that proper appreciation of such drift necessitates a radical reappraisal of our picture of how evolutionary algorithms work (Barnett, 1998; Shipman, Shackleton, Ebner, \& Watson, 2000; Smith, Husbands, Layzell, $\&$ O'Shea, 2002). How does the drift that results from coevolutionary disengagement relate to that experienced by populations percolating across "neutral networks"?

Within the evolutionary computation community, search-space neutrality has been defined as the property of adjacent points in a search space exhibiting equivalent fitness scores. A neutral network is a set of such points, where each member of the set neighbours at least one other member. Clearly, these notions of "adjacency" and "neighbourhood" must be understood in terms of a search algorithm's genetic operators-often this will be a complicated matter. Moreover, the idea of "equivalent fitness" that lies at the heart of the neutrality concept is perhaps not as straightforward as it might appear (see, e.g., Bullock, 2002).

For certain real-world search spaces, such as the RNA folding map, it has been demonstrated that the neutrality present is of a potentially useful kind (Huynen, Stadler, \& Fontana, 1996; Huynen, 1996; Fontana \& Schuster, 1998a, 1998b). Neutrality in this case stems from the fact that many RNA sequences (genotypes) fold into the same secondary structure (phenotypes). This redundancy ensures that some mutations may alter a genotype without altering the associated phenotype. It just so happens that neutral networks percolate the RNA space, ensuring that a large proportion of possible phenotypes (RNA secondary structures) are accessible from an arbitrary genotype via drift. Furthermore, RNA neutral networks enjoy a property of constant innovation in that, over many generations, a neutral walk across such a network will tend to encounter novel phenotypes at a constant rate comparable to that which would be achieved by a random walk in the search space. These properties would seem to ameliorate RNA evolution. Could similar properties be exploited by evolutionary optimization algorithms?

Notice that in this canonical example of neutral drift, the notion of "equivalent fitness" is understood in terms of "equivalent phenotype". Two adjacent RNA strands that fold into the same structure are clearly part of a neutral network-they code for phenotypes that are practically identical. However, since different phenotypes may nevertheless achieve the same fitness score, it is quite possible that a single neutral network may include many different phenotypes that are "selectively neutral" with respect to one another. Although this complication is acknowledged within the neutrality literature, it is not given much attention.

In evolutionary (rather than coevolutionary) systems, if two phenotypes achieve the same fitness score, one has reasonable grounds for classifying them as "equivalent" in some important and enduring sense-selection (ignoring noise and sampling error) will never discriminate between them. However, in a coevolutionary scenario, 
the fact that two phenotypes achieve equivalent fitness is less compelling-since fitness intrinsically depends upon opponents faced, it is entirely possible that they may never achieve equivalent fitness again.

In fact, where fitness is calculated relative to performance against a coevolving population, the notion of neutral drift requires a significant overhaul. No longer can one consider neutral networks to be a (fixed) property of a fitness landscape. Rather, neutrality is now a relational property predicated on the current makeup of both populations. Neutral networks are transient phenomena, merely reflecting the subjective (and hence temporary) equivalences that may exist between the current conspecifics with respect to their current coevolutionary opponents. As a result, whereas neutral drift in an evolutionary system is likely to preserve the quality of evolved solutions by restricting genotypic change such that phenotypes are equivalent in some "objective" sense (e.g., they code for the very same physical structures), the same drift in a coevolutionary system is not necessarily so constrained. In particular, the drift caused by coevolutionary disengagement may involve genotypic changes that correspond to radical phenotypic change, just so long as these phenotypic changes are "undetected" or "invisible" to the individuals that happen to make up the contemporary coevolutionary partner population.

In many cases, given that a disengaged population will often be more likely to generate more disengaged offspring than offspring that re-engage with their coevolutionary partner population, this type of disengaged drift may resemble random movement through the local genetic neighbourhood of the disengaged population. Given that, in the period prior to disengagement, a population contains reasonably high-quality individuals, far from preserving phenotypic quality, this type of disengaged drift is likely to be deleterious.

The exact character of any evolutionary drift is likely to be influenced by the nature of the genetic encodings and genetic operators employed (Bullock, 1999, 2001). Since these encodings and operators may be different for each population involved in a coevolutionary algorithm there is no guarantee that disengaged drift will readily lead to re-engagement. It is possible that encoding/operator biases ensure that drifting populations tend to remain within portions of genotype space that effectively maintain disengagement (as was observed, for example, in the Counting Ones game, section 3.4). In general the issues briefly raised in this section suggest that our appreciation of neutrality and drift and how these phenomena apply to coevolutionary systems deserves more careful consideration.

\section{Future Work \& Conclusions}

Moderate parasite virulence has been implemented throughout this paper with fixed parameter value $\lambda$. However, it is likely that varying $\lambda$ over the course of a run will be necessary in order to drive the system to achieve higher levels of performance (see discussion, section 4.2). While virulence must remain low enough to maintain engagement, it must simultaneously be high enough to encourage progress through imposing strong enough selective pressure. As the system moves through genotype space, the best "compromise" value for $\lambda$ is likely to vary.

For example, in more realistic domains than those employed here, problem asymmetry will not tend to be constant over the course of evolution. Initially one population may enjoy an advantage over the other in terms of the ease with which successful mutant counter-adaptations can be generated. Subsequently, this asymmetry may wax and wane, or even reverse-a population of near-optimal sorting algorithms may enjoy this 
type of advantage over their parasite competitors. Under these conditions, in order to maintain an ideal balance between engagement and selective pressure, $\lambda$ values may need to be constantly varied for each population.

The obvious way to address these concerns is to produce an algorithm that dynamically adapts virulence levels in response to the current state of the coevolutionary system. Whilst a fully autonomous dynamically-adapting virulence algorithm may be difficult to achieve, initial work on a manually-guided virulence algorithm is underway (Bullock, Cartlidge, \& Thompson, 2002). This computational-steering approach allows human controllers to vary the value(s) of $\lambda$ as required during the course of a run. Hopefully, this educational tool will further improve our understanding of engagement and its relationship with virulence.

The problem domains explored in this paper were chosen primarily for their simplicity and the resulting ease of analysis. However, the No Free Lunch theorem (Wolpert \& Macready, 1995) tells us that we should expect the technique presented here to work effectively only on a particular subset of problems. Which problems are good candidates for the approach?

Firstly, the reduced virulence technique is likely to be of use in problems where a reasonable (but not necessarily optimal) solution is required under strong constraints of limited time or computational resources (e.g., dynamic load allocation across a telecommunications network) since reducing parasite virulence can accelerate rapid initial progress towards high-quality solutions despite small population sizes.

Secondly, where the character of the problem is constantly changing and demands constant evolutionary change in the solution population (such as maintaining a strong immune response in a changing environment) reducing virulence is likely to improve performance by encouraging and maintaining engagement.

Finally, for problem spaces where small genetic changes often give rise to qualitative changes at the phenotype level and consequent discontinuous jumps in fitness (e.g., chess strategy) the reduced virulence technique may improve performance by promoting re-engagement when it occurs rather than actively resisting it in the manner of traditional coevolution.

Potentially, coevolutionary algorithms are very valuable and versatile tools, yet there remain an ensemble of problems restricting their successful application. It is our hope that through the introduction of the reduced virulence technique, this paper has contributed to moving coevolutionary computation closer to realising its potential.

\section{References}

Adami, C., Belew, R., Kitano, H., \& Taylor, C. (Eds.). (1998). Artificial Life VI. MIT Press, Cambridge, MA.

Angeline, P. J. (1994). An alternate interpretation of the iterated prisoner's dilemma and the evolution of non-mutual cooperation. In Brooks \& Maes (1994), pp. 353-358.

Angeline, P. J., \& Pollack, J. B. (1993). Competitive environments evolve better solutions for complex tasks. In Forrest, S. (Ed.), Proceedings of the Fifth International Conference on Genetic Algorithms, pp. 264-270. Morgan Kauffmann.

Axelrod, R. (1984). The Evolution of Cooperation. Basic Books, New York.

Barnett, L. (1998). Ruggedness and neutrality — the NKp family of fitness landscapes. In Adami et al. (1998), pp. 18-27.

Barton, N. H., \& Gale, K. S. (1993). Genetic analysis of hybrid zones. In Harrison, R. G. (Ed.), Hybrid Zones and the Evolutionary Process, pp. 13-45. Oxford University Press, Oxford. 
Brooks, R. A., \& Maes, P. (Eds.). (1994). Artificial Life IV. MIT Press, Cambridge, MA.

Bucci, A., \& Pollack, J. (2002). Order-theoretic analysis of coevolution problems: Coevolutionary statics. In Barry, A. M. (Ed.), GECCO 2002 Workshop on Understanding Coevolution: Theory and Analysis of Coevolutionary Algorithms, pp. 229-235.

Bullock, S. (2002). Will selection for mutational robustness significantly retard evolutionary innovation on neutral networks?. In Standish, R., Bedau, M., \& Abbass, H. (Eds.), Artificial Life VIII, pp. 192-201. MIT Press, Cambridge, MA.

Bullock, S. (1999). Are artificial mutation biases unnatural?. In Floreano, D., Nicoud, J.-D., \& Mondada, F. (Eds.), Advances in Artificial Life: Fifth European Conference on Artificial Life (ECAL99), Vol. 1674 of Lecture Notes in Artificial Intelligence, pp. 64-73 EPFL Lausanne, Switzerland. Springer, Berlin.

Bullock, S. (2001). Smooth operator? understanding and visualising mutation bias. In Kelemen, J., \& Sosik, P. (Eds.), Sixth European Conference on Artificial Life (ECAL2001), pp. 602-612 Prague. Springer-Verlag.

Bullock, S., Cartlidge, J., \& Thompson, M. (2002). Prospects for computational steering of evolutionary computation. In et al., E. B. (Ed.), Workshop Proceedings of the Eighth International Conference on Artificial Life, pp. 131-137. MIT Press, Cambridge, MA.

Cartlidge, J., \& Bullock, S. (2002). Learning lessons from the common cold: How reducing parasite virulence improves coevolutionary optimization. In Fogel, D. (Ed.), Congress on Evolutionary Computation, pp. 1420-1425. IEEE Press.

Cliff, D., \& Miller, G. F. (1995). Tracking the Red Queen: Measurements of adaptive progress in co-evolutionary simulations. In Morán, F., Moreno, A., Merelo, J. J., \& Chacón, P. (Eds.), Third European Conference on Artificial Life, Vol. 929 of Lecture Notes in Artificial Intelligence, pp. 200-218. Springer, Berlin.

Combes, C. (1991). Ethological aspects of parasite transmission. The American Naturalist, 138(4).

Davis, L. (Ed.). (1991). Handbook of genetic algorithms. Van Nostrand Reinhold, New York, NY.

Dawkins, R., \& Krebs, J. R. (1979). Arms races between and within species. Proceedings of the Royal Society of London, Series B, 205, 489-511.

De Jong, K. A. (1975). An analysis of the behavior of a class of genetic adaptive systems. Phd thesis, University of Michigan.

Fenner, F., \& Ratcliffe, F. N. (1965). Myxomatosis. Cambridge University Press, Cambridge.

Ficici, S. G., \& Pollack, J. B. (1998a). Challenges in coevolutionary learning: Arms-race dynamics, open-endedness, and mediocre stable states. In Adami et al. (1998), pp. 238-247.

Ficici, S. G., \& Pollack, J. B. (1998b). Coevolving communicative behavior in a linear pursuerevader game. In Pfeifer, R., Blumberg, B., Meyer, J.-A., \& Wilson, S. W. (Eds.), From Animals to Animats 5: Proceedings of the Fifth International Conference on Simulation of Adaptive Behavior, pp. 505-514. MIT Press, Cambridge, MA.

Floreano, D., \& Nolfi, S. (1997). Adaptive behaviour in competing co-evolving species. In Hisbands, P., \& Harvey, I. (Eds.), Fourth European Conference on Artificial Life, pp. 378-387. MIT Press, Cambridge, MA.

Fontana, W., \& Schuster, P. (1998a). Continuity in evolution: On the nature of transitions. Science, $280,1451-1455$.

Fontana, W., \& Schuster, P. (1998b). Shaping space: The possible and the attainable in rna genotype-phenotype mapping. Journal of Theoretical Biology, 194, 491-515.

Futuyma, D. J., \& Slatkin, M. (1983). Coevolution. Sinauer Associates, Sunderland,Mass.

Evolutionary Computation Volume $x$, Number $x$ 
Goldberg, D. E. (1989). Genetic Algorithms in Search, Optimization and Machine Learning. AddisonWesley, Reading, MA.

Goldberg, D. E., \& Richardson, J. (1987). Genetic algorithms with sharing for multimodal function optimization. In Proceedings of the 2nd International Conference on Genetic Algorithms, pp. 41-49. Lawrence Erlbaum Associates.

Hillis, W. D. (1990). Co-evolving parasites improve simulated evolution as an optimization procedure. Physica D, 42, 228-234.

Holland, J. H. (1975). Adaptation in Natural and Artificial Systems. University of Michigan Press, Ann Arbour. Reprinted by MIT Press, 1992.

Hood, G. M. (1997). Spatial structure in a host-pathogen system. In Milliken, P. (Ed.), Frontiers in Ecology: Building the Links. The 1997 Conference of the Ecological Society of Australia, ESA97. Elsevier Science Ltd., Oxford, England.

Husbands, P., \& Mill, F. (1991). Simulated co-evolution as the mechanism for emergent planning and scheduling. In Belew, R. K., \& Booker, L. B. (Eds.), Proceedings of the Fourth International Conference on Genetic Algorithms, pp. 264-270 San Diego, CA. Morgan Kauffman.

Huynen, M. (1996). Exploring phenotype space through neutral evolution. Journal of Molecular Evolution, 43, 165-169.

Huynen, M., Stadler, P., \& Fontana, W. (1996). Smoothness within ruggedness: The role of neutrality in adaption. Proceedings of the National Academy of Science USA, 93(1), 397-401.

Janzen, D. H. (1980). When is it co-evolution?. Evolution, 34(3), 611-612.

Juillé, H. (1995). Incremental co-evolution of organisms: A new approach for optimisation and discovery of strategies. In Morán et al. (1995), pp. 246-260.

Juillé, H., \& Pollack, J. (1998a). Coevolutionary learning: a case study. In Shavlik, J. W. (Ed.), Proceedings of the Fifteenth International Conference on Machine Learning. Morgan Kaufmann.

Juillé, H., \& Pollack, J. (1998b). Coevolving the "ideal" trainer: Application to the discovery of cellular automata rules. In Koza, J. R., Banzhaf, W., Chellapilla, K., Deb, K., Dorigo, M., Fogel, D. B., Garzon, M. H., Goldberg, D. E., Iba, H., \& Riolo, R. (Eds.), Genetic Programming 1998: Proceedings of the Third Annual Conference, pp. 519-527 University of Wisconsin, Madison, Wisconsin, USA. Morgan Kaufmann.

Kauffman, S. A., \& Johnsen, S. (1991). Co-evolution at the edge of chaos: Coupled fitness landscapes, poised states, and co-evolutionary avalanches. Journal of Theoretical Biology, 149(4), $467-505$.

Knuth, D. E. (1973). The Art of Computer Programming - Sorting and Searching, Vol. 3. Addison Wesley.

Land, M., \& Belew, R. K. (1995). No perfect two-state cellular automata for density classification exists. Physical Review Letters, 74(25), 1548-1550.

Levin, B. R. (1996). The evolution and maintenance of virulence in microparasites. Emerging Infectious Diseases, 2(2).

Lindgren, K., \& Nordahl, M. G. (1994). Evolutionary dynamics of spatial games. Physica D, 75, 292-309.

Luke, S., \& Wiegand, R. P. (2002). When coevolutionary algorithms exhibit evolutionary dynamics. In Barry, A. (Ed.), GECCO 2002 Workshop on Understanding Coevolution: Theory and Analysis of Coevolutionary Algorithms, pp. 236-241.

Maes, P., Matarić, M., Meyer, J.-A., Pollack, J., \& Wilson, S. W. (Eds.). (1996). From Animals to Animats 4: Proceedings of the Fourth International Conference on Simulation of Adaptive Behavior. MIT Press, Cambridge, MA. 
Maynard Smith, J. (1989). Evolutionary Genetics. Oxford University Press, Oxford.

Mitchell, M., Crutchfield, J. P., \& Hraber, P. T. (1994). Evolving cellular automata to perform computations: Mechanisms and impediments. Physica D, 75, 361-391.

Morán, F., Moreno, A., Merelo, J. J., \& Chacón, P. (Eds.). (1995). Advances in Artificial Life: Third European Conference on Artificial Life (ECAL'95), Vol. 929 of Lecture Notes in Artificial Intelligence. Springer, Berlin.

Noble, J., \& Watson, R. (2001). Pareto coevolution: Using performance against coevolved opponents in a game as dimensions for pareto selection. In Spencer et al. (2001), pp. 493-500.

Olsson, B. (1996). Optimisation using a host-parasite model with variable-size distributed populations. In Proceedings of the 1996 IEEE International Conference on Evolutionary Computation, pp. 295-299. IEEE Press.

Pagie, L., \& Mitchell, M. (2002). A comparison of evolutionary and coevolutionary search. International Journal of Computational Intelligence and Applications, 2(1), 53-69.

Pagie, L., \& Hogeweg, P. (2000). Information integration and red queen dynamics in coevolutionary optimization. In Proceedings of the 2000 Congress on Evolutionary Computation CEC00, pp. 1260-1267 Piscataway, NJ. IEEE Press.

Paredis, J. (1997). Coevolving cellular automata: Be aware of the red queen!. In Bäch, T. (Ed.), Proceedings of the Seventh International Conference on Genetic Algorithms (ICGA97), pp. 393399 San Francisco, CA. Morgan Kaufmann.

Pollack, J., Blair, A., \& Land, M. (1996). Coevolution of a backgammon player. In Langton, C. G., \& Shimohara, T. (Eds.), Artificial Life $V$ - Proceedings of the Fifth International Workshop on the Synthesis and Simulation of Living Systems Cambridge, MA. MIT Press.

Pollack, J. B., \& Blair, A. D. (1998). Co-evolution in the successful learning of a backgammon strategy. Machine Learning, 32(3), 225-240.

Potter, M. A., \& De Jong, K. A. (1994). A cooperative coevolutionary approach to function optimisation. In Davidor, Y., Schwefel, H.-P., \& Männer, R. (Eds.), Parallel Problem Solving from Nature - PPSN III, pp. 249-257 Berlin. Springer.

Potter, M. A., \& De Jong, K. A. (1995). Evolving neural networks with collaborative species. In Proceedings of the 1995 Summer Computer Simulation Conference, pp. 340-345. The Society of Computer Simulation.

Potter, M. A., \& De Jong, K. A. (2000). Cooperative coevolution: An architecture for evolving coadapted subcomponents. Evolutionary Computation, 8(1), 1-29.

Puppala, N., Sen, S., \& Gordin, M. (1998). Shared memory based cooperative coevolution. In Proceedings of the 1998 IEEE World Congress on Computational Intelligence, pp. 570-574 Anchorage, Alaska, USA. IEEE Press.

Reynolds, C. W. (1994). Competition, coevolution and the game of tag. In Brooks \& Maes (1994), pp. 59-69.

Rosin, C. D. (1997). Coevolutionary search among adversaries. Unpublished phd thesis, University of California, San Diego, Department of Computer Science.

Rosin, C. D., \& Belew, R. K. (1995). Methods for competetive co-evolution: Finding opponents worth beating. In Eshelman, L. J. (Ed.), Proceedings of the Sixth International Conference on Genetic Algorithms, pp. 373-380. Morgan Kaufmann, San Francisco, CA.

Rosin, C. D., \& Belew, R. K. (1997). New methods for competitive coevolution. Evolutionary Computation, 5(1), 1-29. 
Shapiro, J. L. (1998). Does data-model co-evolution improve generalization performance of evolving learners?. In Eiben, A. E., Bäck, T., Schoenauer, M., \& Schwefel, H.-P. (Eds.), Parallel Problem Solving from Nature-PPSN V, Vol. 1498 of Lecture Notes in Computer Science, pp. 540-549 Berlin. Springer.

Shipman, R., Shackleton, M., Ebner, M., \& Watson, R. (2000). Neutral search spaces for artificial evolution: A lesson from life. In Bedau, M. A., McCaskill, J. S., Packard, N. H., \& Rasmussen, S. (Eds.), Artificial Life VII, pp. 162-169. MIT Press, Cambridge, MA.

Sklar, E., \& Pollack, J. (2000). An evolutionary approach to guiding students in an educational game. In Meyer, J.-A., Berthoz, A., Floreano, D., Roitblat, H. L., \& Wilson, S. W. (Eds.), From Animals to Animats 6: Proceedings of the Sixth International Conference on Simulation of Adaptive Behavior, pp. 529-538. MIT Press.

Smith, T. M. C., Husbands, P., Layzell, P., \& O'Shea, M. (2002). Fitness landscapes and evolvability. Evolutionary Computation, 10(1), 1-34.

Spencer, L., Goodman, E. D., Wu, A., Langdon, W., H., Gen, M., Sen, S., Dorigo, M., Pezeshk, S., Garzon, M. H., \& Burke, E. (Eds.). (2001). GECCO 2001: Proceedings of the Genetic and Evolutionary Computation Conference. Morgan Kaufmann.

van Valen, L. (1973). A new evolutionary law. Evolutionary Theory, 1, 1-30.

Watson, R. A., \& Pollack, J. B. (2000). Symbiotic combination as an alternative to sexual recombination in genetic algorithms. In Schoenauer, M., Deb, K., Rudolph, G., Yao, X., Lutton, E., Merelo, J. J., \& Schwefel, H. (Eds.), Parallel Problem Solving from Nature 2000, PPSN VI. Springer-Verlag.

Watson, R. A., \& Pollack, J. B. (2001). Coevolutionary dynamics in a minimal substrate. In Spencer et al. (2001), pp. 702-709.

Wiegand, R. P., Liles, W. C., \& De Jong, K. A. (2001). An empirical analysis of collaboration methods in cooperative coevolutionary algorithms. In Spencer et al. (2001), pp. 1235-1245.

Wolpert, D. H., \& Macready, W. G. (1995). No free lunch theorems for search. Tech. rep. SFI-TR95-02-010, santa fe institute. 